\title{
Otimização da adesão interfacial do compósito fibra de carbono/ epóxi utilizando tratamento superficial oxidativo
}

\author{
Bárbara Silva Sales Guimarães ${ }^{1 *}$ (1) \\ Luiz Eduardo de Carvalho \\ Carlos Alberto Alves Cairo ${ }^{2}$
}

\section{Resumo}

As falhas estruturais em materiais compósitos ocorrem frequentemente devido a problemas no controle das interações interfaciais e o estudo para aprimorar a adesão interfacial entre o reforço e matriz é de suma importância para essa classe de materiais. O objetivo desse trabalho foi otimizar a adesão interfacial do compósito fibra de carbono/epóxi, aplicando um tratamento superficial oxidativo com ácido nítrico no reforço para comparar com compósitos com fibras não tradas. Testes de tração, picnometria, espectroscopia de raios X por dispersão em energia e de microscopia eletrônica de varredura foram usados para avaliar as propriedades e validar o tratamento aplicado. Os resultados obtidos incluem mudanças morfológicas superficiais nas fibras de carbono, alterando a rugosidade e a composição química. Tanto as fibras de carbono 3K quanto as $12 \mathrm{~K}$ após tratamento tiveram um acréscimo de $11 \%$ e $26 \%$ na resistência à tração e também no módulo de elasticidade de $6 \%$ e $16 \%$, respectivamente.

Palavras-chave: Ácido nítrico; Ataque químico; Caracterização morfológica; Melhora da adesão.

\section{Optimization of interfacial adhesion of the fiber carbon/ epoxy composite using surface oxidative treatment}

\begin{abstract}
Structural failures in composite materials often occur due to problems in controlling interfacial interactions and the study to improve the interfacial adhesion between the reinforcement and matriz is of paramount importance for this class of materials. The objective of this work was to optimize the interfacial adhesion of the carbon fiber/epoxy composite, applying oxidative surface treatment with nitric acid in the reinforcement to compare with composites with the untreated fibers. Traction tests, pycnometry, energy dispersion X-ray spectroscopy and scanning electron microscopy were used to determine the properties and validate the treatment applied. The results obtained include superficial morphological changes in the carbon fibers, modifying the roughness and chemical composition. Both the $3 \mathrm{~K}$ and $12 \mathrm{~K}$ carbon fibers after treatment had an increase of $11 \%$ and $26 \%$ in the tensile strength and also in the modulus of elasticity of $6 \%$ and $16 \%$, respectively.
\end{abstract}

Keywords: Nitric acid; Chemical attack; Morphological characterization; Improved adherence.

\section{Introdução}

As fibras de carbono são materiais com ampla aplicabilidade, principalmente como reforço em materiais compósitos utilizados em diversos setores, como, por exemplo, no setor aeroespacial, automobilístico e esportivo. Outras aplicações também começaram a surgir, como em estruturas de máquinas, em lâminas de turbinas e compressores e no setor médico [1].
Dentre as razões para a fibra de carbono ser o reforço mais utilizado, principalmente, em compósitos poliméricos, pode-se citar o mais alto módulo específico e maior resistência específica. Com isso, materiais considerados tradicionais vêm sendo amplamente substituídos por compósitos reforçados com fibras de carbono. Um exemplo dessa substituição é a de ligas de alumínio, que são materiais utilizados em estruturas

${ }^{I}$ Departamento de Engenharia de Materiais, Universidade Tecnológica Federal do Paraná, UTFPR, Londrina, PR, Brasil.

2 Departamento de Ciência e Tecnologia Aeroespacial, Instituto de Aeronáutica e Espaço, ITA, São José dos Campos, SP, Brasil.

*Autor correspondente: barbaraguimaraaes@gmail.com

2176-1523 C 2022. Guimarães et al. Publicado pela ABM. Este é um artigo publicado em acesso aberto (Open Access) sob a licença Creative Commons Attribution, que permite uso, distribuição e reprodução em qualquer meio, sem restrições desde que o trabalho original seja corretamente citado. 
aeronáuticas, que vêm sendo substituídas por compósitos poliméricos estruturais, alcançando uma redução no peso de $20 \%$ a $30 \%$ e, uma redução de $25 \%$ do custo final das peças $[2,3]$.

Quando se discute materiais compósitos um importante segmento de pesquisa é a adesão entre a fibra de carbono e diferentes matrizes poliméricas. A adesão é um dos principais parâmetros para o desempenho estrutural dos compósitos, pois permite a transferência das tensões atuantes da matriz para o reforço através da interface, sendo esta dependente do grau de interações existentes [4-6]. Para que o compósito tenha desempenho mecânico satisfatório é necessária a otimização da adesão interfacial entre seus constituintes [2,7].

Existem alguns tratamentos superficiais para melhorar a adesão interfacial entre o reforço e a matriz, como, por exemplo, oxidação catalítica, deposição química em fase gasosa, tratamentos químicos em fase líquida e enxerto polimérico. Quando é citado tratamentos superficiais em fibras de carbono, os mesmos são divididos entre oxidantes e não oxidantes. Com o tratamento, geralmente são formados sítios químicos ativos, mudanças na rugosidade superficial e outras incorporações/modificações $[4,8]$.

O estudo das técnicas que melhoram a adesão entre a matriz e o reforço nos compósitos é um desafio, pois ainda é pouco desenvolvido principalmente no Brasil. Nesse cenário o presente trabalho teve como objetivo realizar tratamento superficial utilizando uma metodologia de tratamento superficial oxidativo em fase líquida com ácido nítrico para reagir e modificar superfícies de fibras de carbono, para otimizar a adesão do compósito fibra de carbono/ epóxi, alcançando uma melhora no desempenho mecânico estrutural do mesmo. Foi utilizado o tratamento oxidativo em fase liquida por se tratar de um processo contínuo que possibilita a aplicação industrial [4].

As etapas do tratamento oxidativo utilizado neste trabalho foram elaboradas a partir da junção de estudos anteriores [5,6,9-12] que alcançaram modificações efetivas da superfície do tecido de fibra de carbono e obtiveram resultados significativos.

O estudo realizado teve como foco principal a melhoria do desempenho mecânico obtido pelo tratamento superficial oxidativo. Porém, não se considera as propriedades do compósito sem tratamento como não ideais, mas propõe o aumento de propriedades mecânicas utilizando uma combinação comercial de fibra de carbono e matriz epóxi, além de investigar se o tratamento independe do número de filamentos por feixe da fibra.

\section{Metodologia}

Para o desenvolvimento desse trabalho foram utilizados tecidos bidirecionais de fibra de carbono $3 \mathrm{~K}$ (FC 3K) (33MSI) da Hexcel Corporation, tecidos bidirecionais de fibra de carbono $12 \mathrm{~K}$ (FC 12K) adquirida na Barracuda Advanced Composites, resina epóxi 2001 e endurecedor epóxi 3154 da Redelease, e ácido nítrico 68\% P.A da Vetec Química.

Os tecidos de fibra de carbono foram tratados por meio de uma metodologia de tratamento químico superficial oxidativo em fase líquida. Inicialmente foi realizada a limpeza dos tecidos de fibra de carbono utilizando uma lavadora ultrassônica digital da Sanders por 15 minutos em banho de acetona. Essa etapa é essencial para remover da superfície possíveis contaminações, como, por exemplo, gorduras e poeiras. Após a limpeza, ocorreu a secagem em estufa a vácuo à $50^{\circ} \mathrm{C}$ durante 30 minutos $[9,10]$.

$\mathrm{Na}$ segunda etapa, dentro de uma capela foi colocado ácido nítrico em um recipiente de vidro sobre uma chapa de aquecimento (aprox. $100^{\circ} \mathrm{C}$ ). Posteriormente, os tecidos foram imersos no ácido nítrico [3,5,6,9-12].

Após o tratamento químico, as fibras foram lavadas com água destilada e, por último, foi feita a secagem à $50{ }^{\circ} \mathrm{C}$ em estufa à vácuo com pressão de $-600 \mathrm{mmHg}$ por aproximadamente 2 horas $[3,5,6,9]$.

Os tecidos tratados foram utilizados para a fabricação de laminados de matriz epóxi com 4 camadas de tecidos de fibras de carbono, tanto tratadas como sem tratamento. A produção das placas foi feita por laminação em bolsa de vácuo com uso da bomba de vácuo SL-61 Solab, com, aproximadamente, $-600 \mathrm{mmHg}$ de pressão e com duração de 60 minutos em cada uma das combinações de compósitos.

Os primeiros experimentos foram feitos para determinar as densidades relativas dos compósitos por meio da técnica de picnometria.

Os testes de tração foram realizados utilizando a máquina de ensaio universal WDW-100E do modelo DBSL-SJ-10t, com célula de carga de $10000 \mathrm{Kg}$ e distância entre garras de $230 \mathrm{~mm}$. A velocidade da travessa foi de $2 \mathrm{~mm} / \mathrm{min}$. Os ensaios foram realizados baseados na norma ASTMD-3039 [13], com corpos de prova usinados com comprimento de $180 \mathrm{~mm}$, largura de $25 \mathrm{~mm}$ e espessura de $2,0 \mathrm{~mm}$. Os ensaios foram feitos em triplicatas, para cada situação.

Imagens de microscopia eletrônica de varredura das superfícies das fibras de carbono foram analisadas, tanto das fibras sem tratamento como das fibras tratadas. Além disso, imagens das superfícies fraturadas dos corpos de prova após ensaio de tração foram também analisadas. Análises de elementos químicos foram feitas com acoplamento da espectroscopia de raios $\mathrm{X}$ por energia dispersiva com aplicação de $25 \mathrm{kV}$ no equipamento de varredura FEI Inspect S 50. Utilizando essas técnicas, foram feitos estudos investigativos de mudanças na morfologia da superfície das fibras de carbono, comparando por meio das imagens das fibras de carbono sem tratamento e após tratamento químico. A partir das imagens das superfícies fraturadas foi investigado a presença de resina aderida nas fibras após a fratura. Já a análise química da superfície foi feita para analisar o teor (em \%) dos elementos químicos, como, o oxigênio, carbono e nitrogênio, antes e depois do tratamento. Com essas análises buscou-se a confirmação do aumento 
em propriedades mecânicas com a alteração na adesão fibra/ matriz por meio do processo oxidativo superficial da fibra da efetivação do tratamento químico proposto neste trabalho.

\section{Resultados e Discussão}

\subsection{Massa específica dos componentes do compósito}

Utilizando a técnica de picnometria foi determinado a densidade relativa dos compósitos produzidos. A partir dos resultados obtidos, que estão apresentados na Tabela 1 , nota-se que os valores das massas específicas dos compósitos que foram produzidos com fibras de carbono tratadas quimicamente possuem valores maiores do que os compósitos produzidos com fibras de carbono não tratadas. Este é o primeiro indício de uma melhora na adesão interfacial, já que compósitos mais compactos possuem valores de densidade maiores.

Essa adesão pode ter ocorrido pela formação de sulcos(ranhuras), pela formação de novos grupos químicos superficiais compatíveis com a resina epóxi, pelo aumento da rugosidade superficial criada pelo tratamento, ou pela junção desses fatores.

\subsection{Frações volumétricas}

Com os valores das densidades das fibras de carbono e da matriz epóxi, encontrados nos data sheets, de acordo com os fornecedores e, com os valores das massas dos reforços, das matrizes e dos compósitos que foram medidos durante processo de produção, foi possível obter os valores das frações volumétricas de cada um dos constituintes do compósito e determinado o valor da fração volumétrica de vazios. Apenas foi possível determinar esses valores para compósitos produzidos com fibras de carbono sem tratamento, pois não foram tiradas medidas de densidade das fibras tratadas.

$\mathrm{O}$ volume de vazios $\left(\mathrm{v}_{\mathrm{v}}\right)$ foi determinado utilizando a Equação 1, sendo $\mathrm{v}_{\mathrm{T}} \mathrm{v}_{\mathrm{F}}$ e $\mathrm{v}_{\mathrm{M}}$ o volume do compósito, do reforço e da matriz, respectivamente. Calculou-se as frações volumétricas de reforço $\left(\mathrm{V}_{\mathrm{F}}\right)$, da matriz $\left(\mathrm{V}_{\mathrm{M}}\right)$ e de vazios $\left(\mathrm{V}_{\mathrm{V}}\right)$ utilizando a Equação 2 [4].

$$
\begin{aligned}
& \mathrm{VT}=\mathrm{VF}+\mathrm{VM}+\mathrm{Vv}_{\mathrm{V}} \\
& \mathrm{V}_{\mathrm{F}}=\frac{\mathrm{v}_{\mathrm{F}}}{\mathrm{v}_{\mathrm{T}}} ; \mathrm{V}_{\mathrm{M}}=\frac{\mathrm{v}_{\mathrm{M}}}{\mathrm{v}_{\mathrm{T}}} ; \mathrm{V}_{\mathrm{V}}=\frac{\mathrm{v}_{\mathrm{V}}}{\mathrm{v}_{\mathrm{T}}}
\end{aligned}
$$

Os valores calculados podem ser observados na Tabela 2. Observa-se que, no caso dos compósitos com fibras de carbono, tanto com $3 \mathrm{~K}$ quanto os que continham fibras $12 \mathrm{~K}$, as frações volumétricas de vazios ficaram maiores do que o esperado (esperava-se valores menores do que $5 \%$ ), e as frações volumétricas das fibras de carbono ficaram abaixo do esperado (esperava-se frações de aproximadamente 50\%).
Esses resultados podem ser consequência da ineficiência do processo de aplicação de vácuo, resultando em compósitos com alta fração volumétrica de vazios e de matriz [4].

\subsection{Análise química}

Espectroscopia de raio X por dispersão em energia nas fibras de carbono tratadas e não tratadas foi utilizada para determinar mudanças nas percentagens de elementos químicos na superfície das fibras de carbono.

As quantidades dos elementos de carbono, nitrogênio e oxigênio estão mostrados na Tabela 3. Foram analisadas

Tabela 1. Comparativo das massas específicas dos compósitos fabricados com as fibras $3 \mathrm{~K}$ e $12 \mathrm{~K}$, conforme recebidas e com tratamento oxidativo

\begin{tabular}{cc}
\hline Compósito & Massa específica $\left(\mathbf{g} / \mathbf{c m}^{3}\right)$ \\
\hline FC 3K Sem Tratamento & 1,18 \\
FC 3K Tratadas & 1,34 \\
FC 12K Sem Tratamento & 1,17 \\
FC 12K Tratadas & 1,21 \\
\hline
\end{tabular}

Tabela 2. Frações volumétricas para os compósitos obtidos sem tratamento oxidativo

\begin{tabular}{cc}
\hline Compósitos & Frações Volumétricas (V) (\%) \\
\hline FC 3K Sem Tratamento & $\mathrm{V}_{\mathrm{F}}=23,47$ \\
& $\mathrm{~V}_{\mathrm{M}}=68,98$ \\
$\mathrm{~V}_{\mathrm{v}}=7,55$ \\
\\
FC 12K Sem Tratamento & $\mathrm{V}_{\mathrm{F}}=23,31$ \\
& $\mathrm{~V}_{\mathrm{M}}=68,75$ \\
& $\mathrm{~V}_{\mathrm{V}}=7,94$ \\
\hline
\end{tabular}

Tabela 3. Variação da quantidade dos elementos Carbono (C), Nitrogênio $(\mathrm{N})$ e Oxigênio $(\mathrm{O})$ na superfície das fibras de carbono

\begin{tabular}{|c|c|c|c|c|c|c|}
\hline \multirow{2}{*}{ Fibra } & \multirow{2}{*}{ Região } & \multirow{2}{*}{ Elemento } & \multicolumn{2}{|c|}{$\begin{array}{c}\text { FC Sem } \\
\text { Tratamento }\end{array}$} & \multicolumn{2}{|c|}{ FC Tratadas } \\
\hline & & & $\begin{array}{c}\text { Peso } \\
(\%)\end{array}$ & $\begin{array}{c}\text { Atômico } \\
(\%)\end{array}$ & $\begin{array}{c}\text { Peso } \\
(\%)\end{array}$ & $\begin{array}{c}\text { Atômico } \\
(\%)\end{array}$ \\
\hline \multirow[t]{9}{*}{$3 \mathrm{~K}$} & $\mathrm{~A}$ & $\mathrm{C}$ & 93,49 & 94,55 & 93,01 & 94,30 \\
\hline & & $\mathrm{N}$ & 4,67 & 4,05 & 3,55 & 3,09 \\
\hline & & $\mathrm{O}$ & 1,84 & 1,40 & 3,43 & 2,61 \\
\hline & B & $\mathrm{C}$ & 90,02 & 91,59 & 88,63 & 90,42 \\
\hline & & $\mathrm{N}$ & 7,27 & 6,34 & 8,01 & 7,00 \\
\hline & & $\mathrm{O}$ & 2,71 & 2,07 & 3,36 & 2,57 \\
\hline & $\mathrm{C}$ & $\mathrm{C}$ & 92,12 & 93,40 & 82,91 & 85,85 \\
\hline & & $\mathrm{N}$ & 5,56 & 4,83 & 7,84 & 6,96 \\
\hline & & $\mathrm{O}$ & 2,33 & 1,77 & 9,26 & 7,20 \\
\hline \multirow[t]{9}{*}{$12 \mathrm{~K}$} & A & $\mathrm{C}$ & 93,98 & 94,91 & 91,53 & 92,87 \\
\hline & & $\mathrm{N}$ & 4,80 & 4,15 & 6,23 & 5,42 \\
\hline & & $\mathrm{O}$ & 1,23 & 0,93 & 2,24 & 1,70 \\
\hline & B & $\mathrm{C}$ & 93,24 & 94,27 & 95,27 & 96,02 \\
\hline & & $\mathrm{N}$ & 5,60 & 4,85 & 3,79 & 3,28 \\
\hline & & $\mathrm{O}$ & 1,16 & 0,88 & 0,94 & 0,71 \\
\hline & $\mathrm{C}$ & $\mathrm{C}$ & 91,42 & 92,78 & 90,47 & 91,93 \\
\hline & & $\mathrm{N}$ & 6,34 & 5,52 & 7,32 & 6,38 \\
\hline & & $\mathrm{O}$ & 2,24 & 1,70 & 2,22 & 1,69 \\
\hline
\end{tabular}
$3 \mathrm{~K}$ e $12 \mathrm{~K}$ tradas e não tratadas 
três regiões diferentes na superfície das fibras de carbono, analisando a percentagem em peso e atômica dos elementos químicos carbono, oxigênio e nitrogênio. Pode-se observar que, com algumas pouquíssimas exceções, as mudanças nas \% dos elementos, nas três regiões de todas as fibras de carbono analisadas, apresentaram comportamentos similares, onde a percentagem de carbono diminui, e ocorreu um aumento de nitrogênio e oxigênio nos dois tipos (FC 3K e FC 12K) após o tratamento químico. Esse resultado também valida a efetividade do tratamento químico (oxidativo) dessa pesquisa, já que o ácido nítrico é composto basicamente por oxigênio e nitrogênio e, esses elementos, durante tratamento na superfície das fibras de carbono interagem com grupos químicos da mesma.

\subsection{Microscopia eletrônica de varredura}

Na Figura 1 são apresentadas imagens obtidas por microscopia eletrônica de varredura por elétrons secundários,

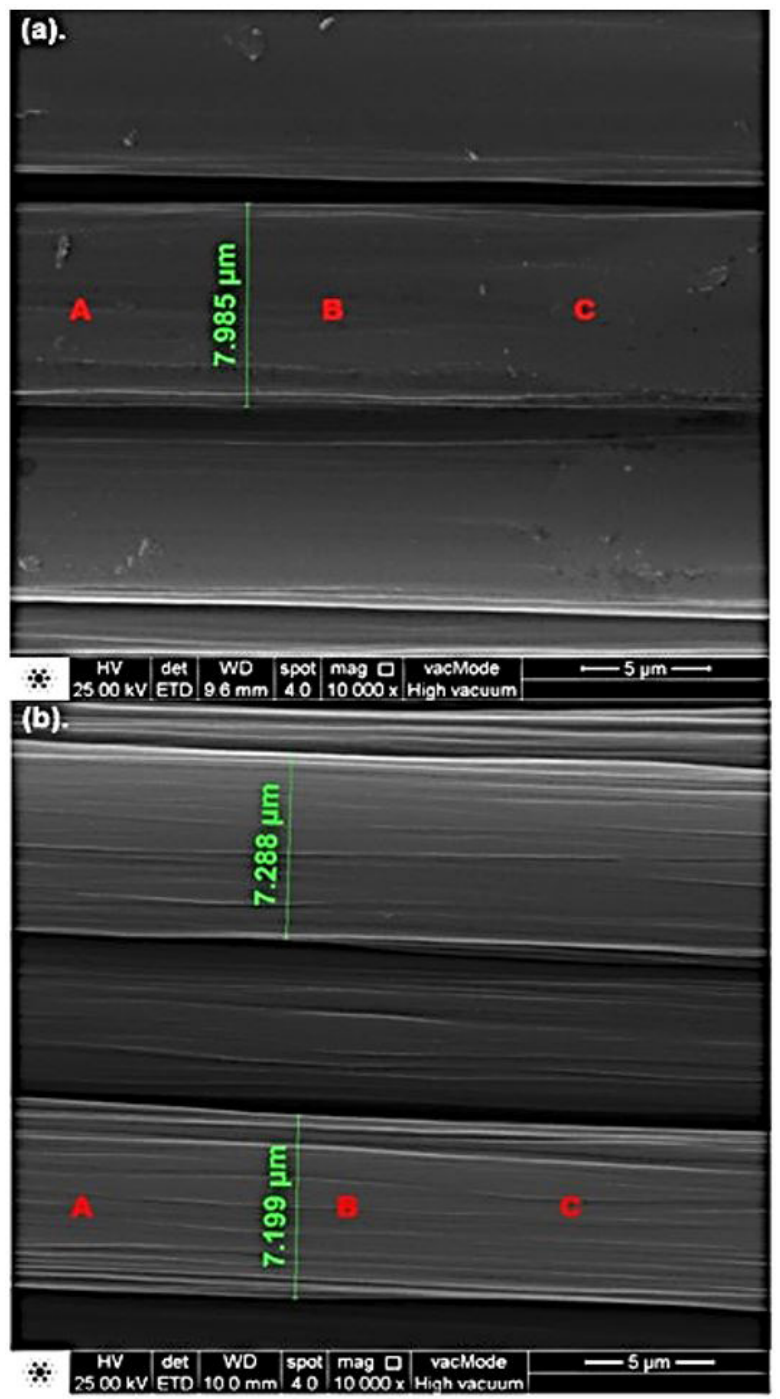

Figura 1. Microscopia Eletrônica de Varredura: (a). regiões A, B e C das FC 3K sem tratamento; (b) regiões A, B e C da FC 3K tratadas. que foram feitas em 3 regiões distintas nos dois tipos de fibras de carbono, com tratamento e sem tratamento. Observa-se que nas $\mathrm{FC} 3 \mathrm{~K}$ que foram tratadas quimicamente existem formações de sulcos na superfície das fibras nas três regiões de forma homogênea. Esses sulcos indicam aumento na rugosidade superficial das fibras e, consequentemente, favorece um aumento na adesão interfacial entre matriz e o reforço nos compósitos.

Foi observado tanto nas FC $3 \mathrm{~K}$ sem tratamento (Figura 1a) quanto nas FC 12K sem tratamento (Figura 2a) a existência de pequenos resíduos. No caso das FC $3 \mathrm{~K}$, esses resíduos foram totalmente eliminados após a etapa da limpeza no processo de tratamento oxidativo (Figura 1b) e, em relação as FC 12K, pode-se observar a existência de menor quantidade de resíduos após a etapa da limpeza (Figura 2b).

Na Figura 2 são apresentadas as micrografias da superfície das FC $12 \mathrm{~K}$ sem tratamento e depois de serem tratadas. Pode-se observar que não apresentaram uma mudança significativa em relação a sua morfologia. Com a
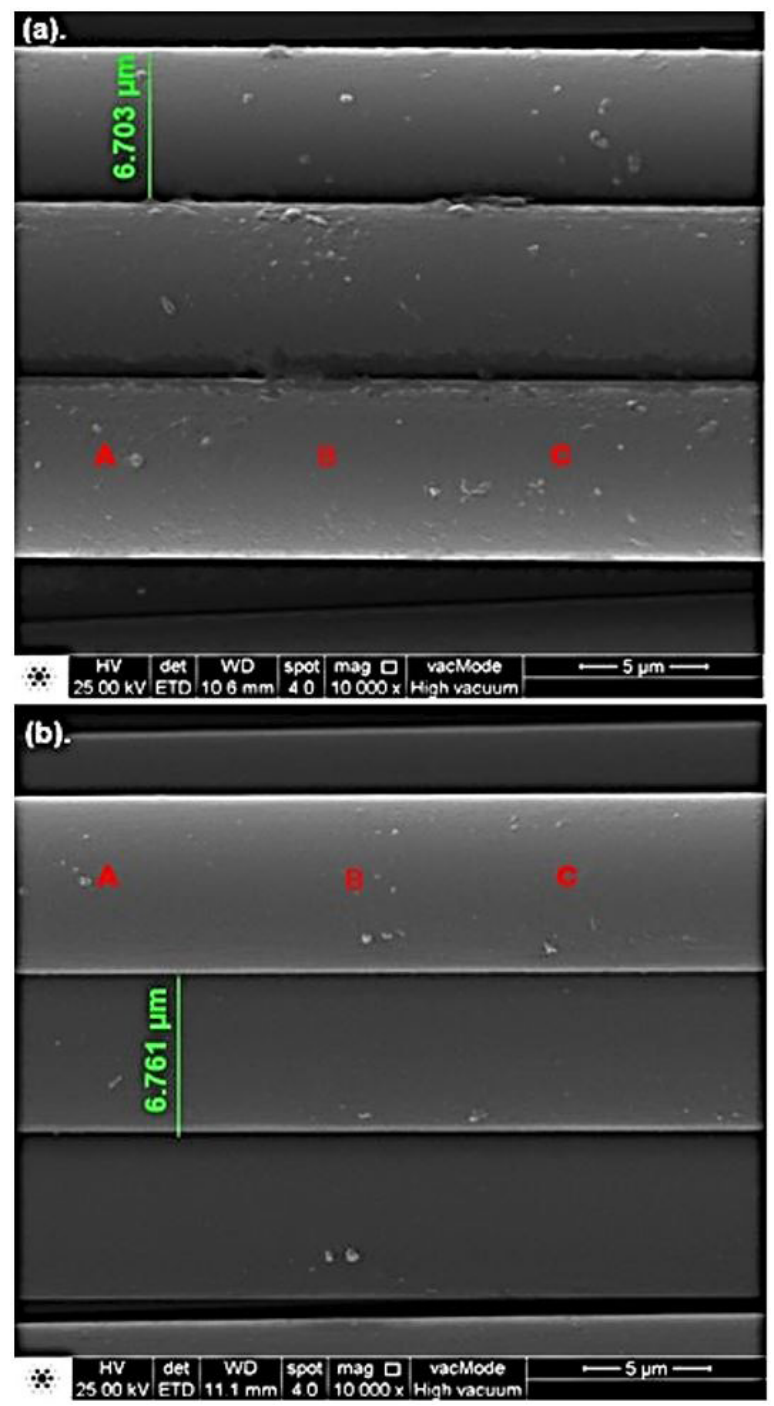

Figura 2. Microscopia Eletrônica de Varredura: (a). regiões A, B e C das FC 12K sem tratamento; (b) regiões A, B e C da FC 12K tratadas. 
retirada dos resíduos pode-se ter a impressão de que as fibras apresentam um aspecto mais liso. Esse fato pode ter ocorrido devido há alguns fatores como: tempo insuficiente de tratamento para essa fibra específica ou a existência de um recobrimento na superfície das fibras diminuindo a efetividade do ataque químico. $\mathrm{O}$ tempo não foi aumentado neste estudo para permitir a comparação com uma única variável, a quantidade de filamentos ( $3 \mathrm{~K}$ e $12 \mathrm{~K}$ ).

As mudanças morfológicas das FC $3 \mathrm{~K}$ são características da efetividade do tratamento superficial proposto. Em comparação, nas FC 12K não foram observadas mudanças significativas. Sabendo que o processo oxidativo, nos dois casos, foi feito da mesma maneira, é demonstrado que o número de filamentos por feixe pode ter dificultado o acesso da solução em toda a superfície de todos os monofilamentos que compõe o feixe (FC 12K).

As imagens das FC 3K fraturadas estão apresentadas na Figura 3. Observa-se pelas imagens das fibras tratadas quimicamente (Figura $3 \mathrm{~b}$ e Figura $3 \mathrm{~d}$ ) que as mesmas possuem mais resina aderida do que as fibras fraturadas que não possuíam tratamento químico (Figura 3a e Figura 3 c). A resina aderida nas fibras após fratura está indicada pelas setas vermelhas. Além deste indicativo, pode-se observar que existe quantitativamente mais resíduo de resina aderida nas FC $3 \mathrm{~K}$ tratadas do que nas FC $3 \mathrm{~K}$ sem tratamento. A presença de resíduos de resina na superfície das fibras após fratura indica uma melhor interação fibra-matriz, resultando assim em uma melhor adesão. $\mathrm{O}$ aspecto mais liso das fibras de carbono sem tratamento é um indicativo de que a falha ocorreu na interface entre fibra e matriz por descolamento, indicando assim uma adesão interfacial mais fraca. As mudanças morfológicas das FC $3 \mathrm{~K}$ são características da efetividade do tratamento superficial proposto.

Observando a Figura 4, onde são mostradas FC 12K, com e sem tratamento, não é possível afirmar a efetividade do processo oxidativo, não sendo observado mudança significativa na quantidade de resina aderida na superfície da fibra, apresentando apenas um singelo incremento nesta quantidade.

\subsection{Comportamento mecânico}

Na Figura 5 são apresentadas as curvas tensão $\mathrm{X}$ deformação que representam comparativos entre o

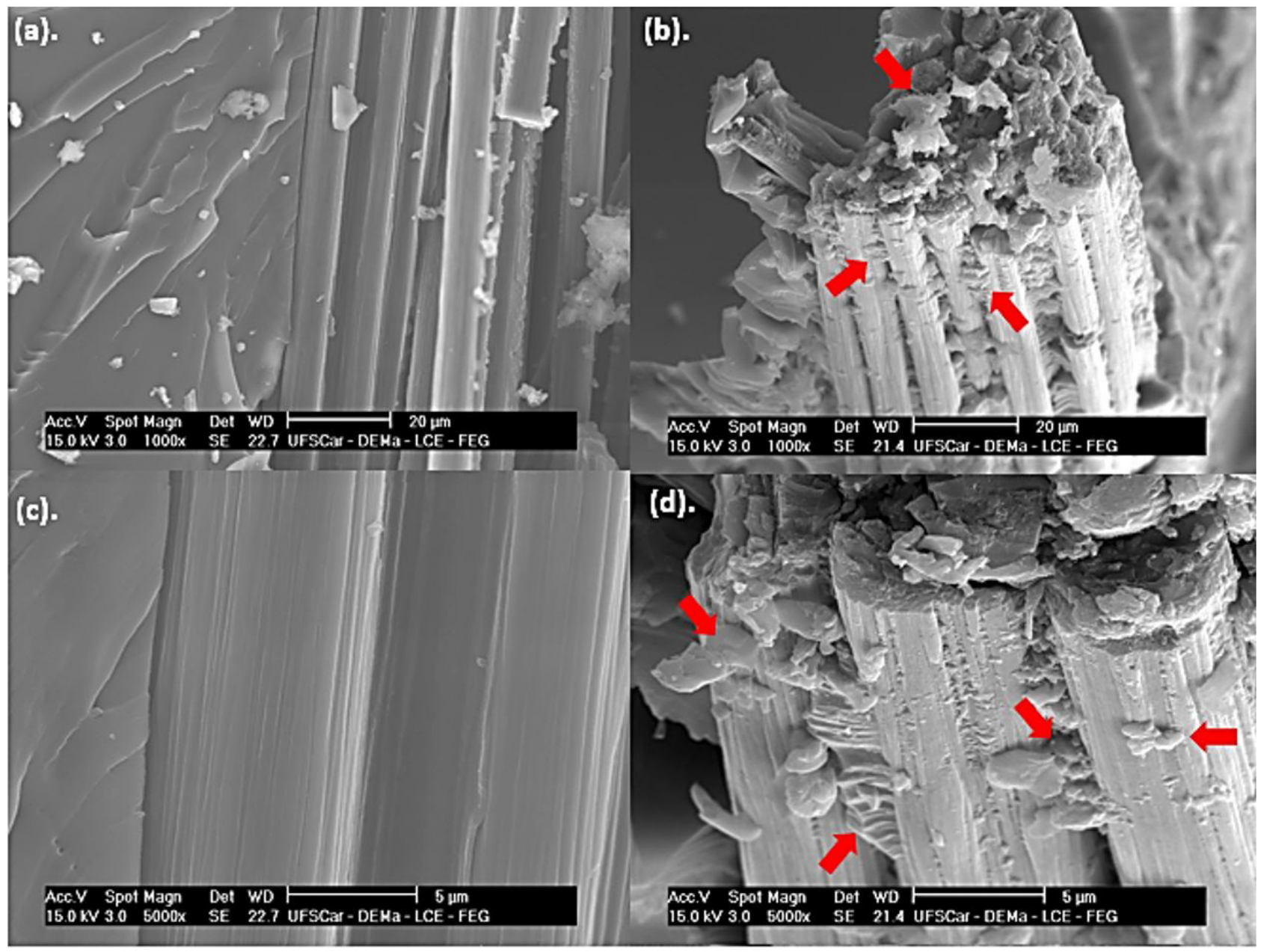

Figura 3. Microscopia Eletrônica de Varredura em FC 3K: (a) sem tratamento em escala de $20 \mu \mathrm{m}$ e (b) com tratamento em escala de $20 \mu \mathrm{m}$; (c) sem tratamento em escala de $5 \mu \mathrm{m}$ e (d) com tratamento em escala de $5 \mu \mathrm{m}$. 


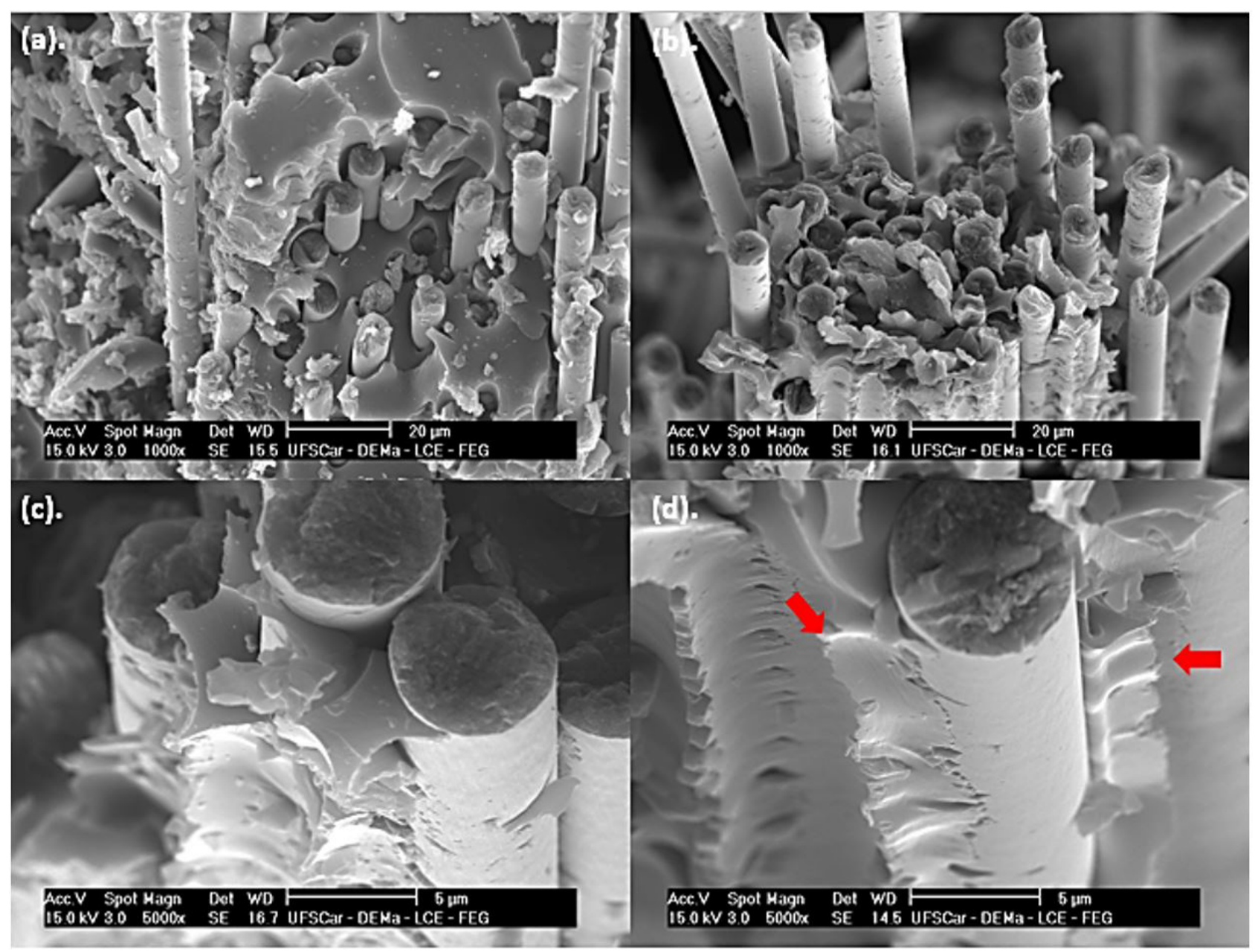

Figura 4. Microscopia Eletrônica de Varredura em FC 12K: (a) sem tratamento em escala de $20 \mu \mathrm{m}$ e (b) com tratamento em escala de $20 \mu \mathrm{m}$; (c) sem tratamento em escala de $5 \mu \mathrm{m}$ e (d) com tratamento em escala de $5 \mu \mathrm{m}$.

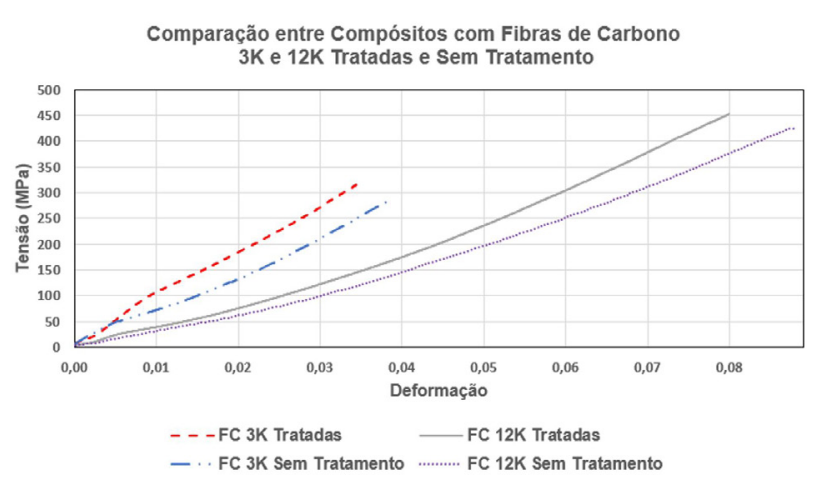

Figura 5. Comportamento Tensão x Deformação de Compósitos com FC $3 \mathrm{~K}$ e $12 \mathrm{~K}$ Tratadas e Sem Tratamento.

comportamento mecânico dos compósitos que utilizaram FC $3 \mathrm{~K}$ e $12 \mathrm{~K}$ sem tratamento químico e para as FC $3 \mathrm{~K}$ e $12 \mathrm{~K}$ com tratamento químico, totalizando 4 combinações diferentes. A Tabela 4apresenta resultados de três propriedades mecânicas, sendo, resistência à tração, módulo elástico e deformação de ruptura, de cada um dos 4 tipos diferentes de compósitos ensaiados.
Tabela 4. Propriedades Mecânicas dos compósitos com fibras de carbono com $3 \mathrm{~K}$ e $12 \mathrm{~K}$ filamentos

\begin{tabular}{cccc}
\hline Compósito & $\begin{array}{c}\text { Limite de } \\
\text { Resistência à } \\
\text { Tração (MPa) }\end{array}$ & $\begin{array}{c}\text { Módulo } \\
\text { Elástico (GPa) }\end{array}$ & $\begin{array}{c}\text { Deformação } \\
\text { na ruptura } \\
\mathbf{( \% )}\end{array}$ \\
\hline $\begin{array}{c}\text { FC 3K Sem } \\
\text { tratamento } \\
\text { FC 3K }\end{array}$ & 284 & 6,93 & 3,84 \\
$\begin{array}{c}\text { Tratadas } \\
\text { FC 12K Sem } \\
\text { tratamento } \\
\text { FC 12K }\end{array}$ & 415 & 8,75 & 3,45 \\
Tratadas & 426 & 4,90 & 8,80 \\
\hline
\end{tabular}

Primeiramente, analisando os compósitos com FC $3 \mathrm{~K}$, observa-se que a resistência à tração apresentou um aumento de aproximadamente $11 \% \mathrm{e}$, um aumento de $26 \%$ no módulo elástico nos compósitos que usaram fibras de carbono com tratamento químico. O aumento no módulo elástico é uma consequência direta do aumento da adesão pois, quanto mais interconectada a interface entre matriz e reforço, mais rígido será o compósito. 
Em seguida, observa-se que os compósitos reforçados com FC $12 \mathrm{~K}$ tratadas obtiveram um aumento de $16 \%$ no módulo elástico e um aumento de aproximadamente $6 \%$ na resistência à tração, mesmo essas não tendo mudanças significativas em relação a sua morfologia superficial por meio da análise de microscopia eletrônica de varredura, tanto nas imagens da superfície das fibras e das fibras fraturadas.

Em relação aos valores de deformações de ruptura, apresentados na Tabela 4, os compósitos que continham FC $\mathrm{K}$ possuem valores bem menores do que os valores dos compósitos com FC 12K. Este fato tem como relação o módulo elástico, pois, quanto maior o módulo elástico, menor é a deformação de ruptura. $\mathrm{O}$ decréscimo nos valores de deformação de ruptura nos compósitos que utilizaram as fibras tratadas, nos dois casos, ocorreu por consequência de uma maior rigidez causada pela melhora na adesão interfacial da matriz com o reforço dos compósitos.

Esses resultados junto com os resultados já descritos anteriormente em outras análises são significativos e, mostram a efetividade do tratamento superficial das fibras com ácido nítrico para melhorar a adesão interfacial entre reforço e matriz.

Por último, é importante relatar que os resultados do comportamento mecânico dos compósitos estão muito abaixo dos valores conhecidos para compósitos de matriz epóxi com reforço bidirecional de fibra de carbono. Esses resultados são consequências das baixas frações volumétricas de fibras e pelas altas frações volumétricas de vazios nos compósitos, que podem ser melhoradas utilizando outros métodos de produção, como, por exemplo, moldagem em autoclaves $[2,4]$.

\section{Conclusão}

O estudo da otimização da adesão foi inicialmente feito por meio da técnica de picnometria, onde foi possível determinar que os compósitos que continham como reforço fibras de carbono tratadas pelo processo oxidativo apresentaram valores superiores de massa específica do que os compósitos com fibras de carbono sem tratamento.

Por meio do estudo de imagens de microscopia eletrônica de varredura observou-se mudanças nas morfologias das fibras tratadas, como, formações de sulcos superficiais para melhor ancoramento mecânico pela resina e limpezas efetivas para a homogeneização superficial. Além disso, as imagens das superfícies das FC $3 \mathrm{~K}$ tratadas após fratura do compósito apresentam aumento na quantidade de matriz aglutinada nas fibras do que nas fibras que não foram tratadas, sendo também um indicativo de melhora na adesão interfacial matriz-reforço.

Resultados das análises químicas confirmaram, na média, o aumento na percentagem dos elementos oxigênio e nitrogênio e, reduções nos valores percentuais de carbono na superfície das fibras. Por último, a comprovação da efetividade do tratamento químico proposto foi feita por meio do estudo do comportamento mecânico dos compósitos. Os valores do limite de resistência à tração e do módulo elástico aumentaram significativamente para os compósitos com reforços de fibras de carbono tratadas, e ocorreram decréscimos nos valores de deformação de ruptura desses mesmos compósitos por consequência de uma maior rigidez. Estes fatos são decorrentes da melhora na adesão interfacial da matriz-reforço dos compósitos.

Os baixos valores do limite de resistências, considerando que se trata de compósitos fibra de carbono/epóxi, são decorrentes das baixas frações volumétricas de fibras e das altas frações volumétricas de vazios nos compósitos, calculadas a partir das densidades encontradas pela técnica de picnometria.

Concluindo, ocorreram alterações físicas e químicas nas superfícies das fibras de carbono após o tratamento oxidativo superficial utilizando ácido nítrico e, como consequência, a adesão interfacial entre a matriz epóxi e o reforço de fibras de carbono foi otimizada, aumentando significativamente as propriedades mecânicas de limite de resistência à tração e módulo elástico dos laminados ensaiados.

\section{Referências}

1 Chawla KK. Composite materials: science and engineering. 3. ed. Birmingham: Springer; 2011. 533 p.

2 Callister WD Jr. Ciência e engenharia de materiais: uma introdução. 7. ed. Rio de Janeiro:LTC Editora; 2008. 704 p.

3 Rezende MC, Costa ML, Botelho EC. Compósitos estruturais: tecnologia e prática. São Paulo: Artliber Editora Ltda; 2011.396 p.

4 Levy F No. Pardini LC. Compósitos estruturais: ciência e tecnologia. São Paulo: Edgard Blucher; 2006. 313 p.

5 Burakowski L, Rezende MC. Modificação da rugosidade de fibras de carbono por método químico para aplicação em compósitos poliméricos. Polímeros, Ciência e Tecnologia. 2001;11(2):51-57.

6 Woodhead AL, Souza ML, Church JS. An investigation into the surface heterogeneity of nitric acid oxidized carbon fiber. Applied Surface Science. 2017;401:79-88.

7 Tita V. Projeto e fabricação de estruturas em materiais compósito polimérico. São Carlos: USP; 2007. 68 p.

8 Lubin G. Handbook of composites. Nova York: Van Nostrand Reinhold; 1982.786 p. 
9 Xu B, Wang X, Lu Y. Surface modification of polyacrylonitrile-based carbon fiber and its interaction with imide. Applied Surface Science. 2006;253(30):2695-2701.

10 Yuan H, Wang C, Zhang S, Lin X. Effect of surface modification on carbon fiber and its reinforced phenolic matrix composite. Applied Surface Science. 2006;259:288-293.

11 Wu S, Liu Y, Ge Y, Ran L, Peng K, Yi M. Surface structures of PAN-based carbon fibers and their influences on the interface formation and mechanical properties of carbon-carbon composites. Composites Parte A: AppliedScieceand Manufacturing. 2016;90:480-488.

12 Zhang J, Tang H, Hao S. Study on Carbon Fiber Surface Treatment Based on Surface Oxidation Method. In: Proceedings of the $7^{\text {th }}$ International Forum on Strategic Technology (IFOST); 2012; Tomsk, Rússia. USA: IEEE; 2013. p. 327-345.

13 ASTM International. ASTM D3039/D3039 M-00. Standard Teste Method for Tensile Properties of Polymer Matrix Composite Meterials. West Conshohocken, PA: ASTM International; 2000.

Recebido em: 25 Mar. 2021

Aceito em: 05 Ago. 2021 